\title{
A Randomized Controlled Trial of an Internet-Delivered Treatment: Its Potential as a Low-Intensity Community Intervention for Adults With Symptoms of Depression
}

Derek Richards ${ }^{1,2}$, M Sc,, PhD; Ladislav Timulak ${ }^{2}$, , PhD; Emma O'Brien ${ }^{1}$, MSc (Applied Psychol; Claire Hayes ${ }^{3}$, Psy.D.; Noemi Vigano ${ }^{1}$, MSc (Psych); John Sharry ${ }^{4}$, PhD; Doherty Gavin ${ }^{5}$, D Phil

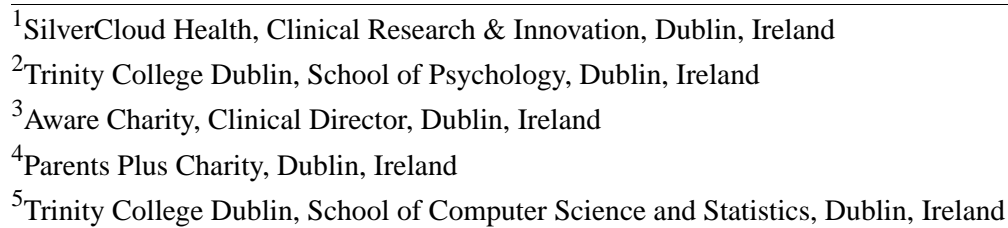

\section{Corresponding Author:}

Derek Richards, M Sc,, $\mathrm{PhD}$

SilverCloud Health

Clinical Research \& Innovation

The Priory, Johns St West, Dublin 8

Dublin,

Ireland

Phone: 3530867965581

Fax: 3530867965581

Email: drichard@tcd.ie

\section{Abstract}

Background: Online delivered treatments for depression have proved successful, with supported programs offering the potential for improved adherence and outcomes. Online support is particularly interesting in the context of increasing access to interventions, and delivering interventions population-wide.

Objective: The research investigated if a supported online treatment is effective as a low-intensity community-based intervention for adults with depression. Based on previous successes with supported online treatments for, and the specific Space from Depression program, it was hypothesized that participants in the trial would demonstrate significant decreases in depressive symptoms post-intervention, with corresponding improvements in comorbid anxiety and quality of life indicators.

Methods: The study was a randomized controlled trial of a supported 8 module online cognitive behavioral therapy (iCBT) program for adults with depressive symptoms $(n=96)$ compared to a waiting-list control group $(n=92)$. The primary outcome was depressive symptoms. The program was made available nationwide using trained supporters from an established depression charity.

Results: For the treatment group, post-treatment effect sizes reported were large for the primary outcome measure on depression $(d=1.19)$. The between-group effects were moderate for the primary outcomes $(d=0.40)$ favoring the treatment group. Gains were maintained at 6-month follow-up.

Conclusions: The results from the present study show that the SilverCloud internet-delivered cognitive-behavior therapy (iCBT) program, Space from Depression, is effective in reducing depressive symptoms in comparison to a waiting list control group. The study demonstrated the potential of an online delivered treatment with online support in a community sample of Irish adults 'with symptoms of depression. It gives support to a model for delivering online depression interventions population-wide using trained supporters. In locations where behavioral and mental health services are underdeveloped, or where structures simply do not exist, or where there is a potential to offset risk and escalation of difficulties and benefit from early intervention, such a model of service provision could be feasible.

Trial Registration: International Standard Randomized Controlled Trial Number (ISRCTN): 03704676; http://www.controlled-trials.com/ISRCTN03704676 (Archived by WebCite at http://www.webcitation/6cWQZmEvw). 
(iProc 2015;1(1):e5) doi:10.2196/iproc.4679

\section{KEYWORDS}

CBT; depression; Internet delivered; online interventions; population health; randomized trial; symptoms; treatment

(This is a conference paper presented at the Connected Health Symposium, Boston, 2015, which was not edited and is only lightly peer-reviewed)

\section{Multimedia Appendix 1}

Extended abstract.

[PDF File (Adobe PDF File), 487KB - iproc_v1i1e5_app1.pdf ]

\section{References}

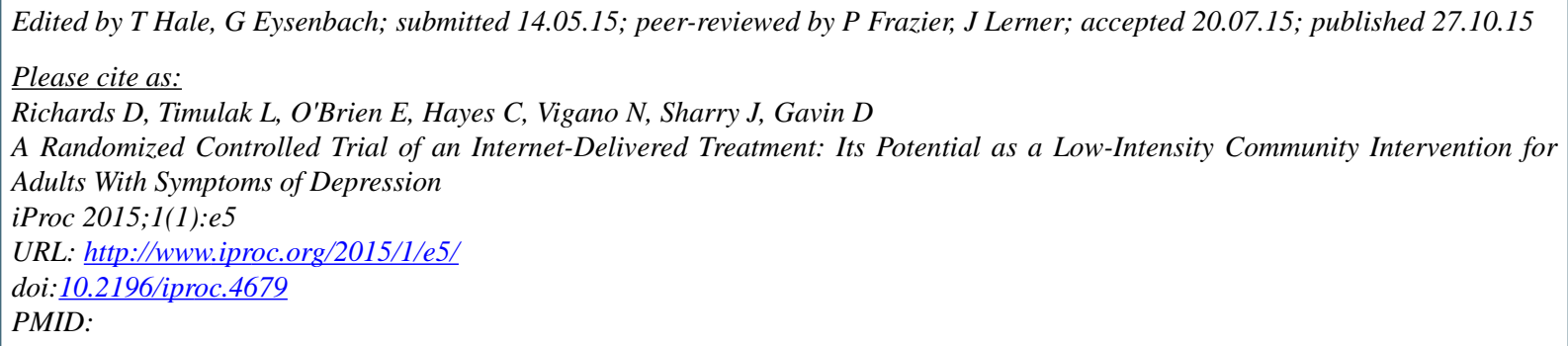

CDerek Richards, Ladislav Timulak, Emma O'Brien, Claire Hayes, Noemi Vigano, John Sharry, Doherty Gavin. Originally published in JMIR Mhealth and Uhealth (http://www.iproc.org), 27.10.2015. This is an open-access article distributed under the terms of the Creative Commons Attribution License (http://creativecommons.org/licenses/by/2.0/), which permits unrestricted use, distribution, and reproduction in any medium, provided the original work, first published in JMIR mhealth and uhealth, is properly cited. The complete bibliographic information, a link to the original publication on http://mhealth.jmir.org/, as well as this copyright and license information must be included. 